\title{
For Organizational Communication that Promotes the Sharing of Tacit Knowledge
}

\author{
Márcio Oliveira ${ }^{1}$ \\ ${ }^{1}$ NECE - Research Center in Business Sciences of the University of Beira Interior, Politécnico de Leiria, Portugal \\ Correspondence: Márcio José Sol Pereira Oliveira, Rua General Norton de Matos, Apartado 4133, 2411-901 Leiria, \\ Portugal. E-mail: marcio.oliveira@ipleiria.pt
}

Received: December 3, 2020 Accepted: December 14, 2020 Online Published: December 15, 2020

\begin{abstract}
Objectives - focusing the study's focus on sharing tacit knowledge in non-profit organizations (NPOs) in Portugal and taking as a case study the Portuguese volunteer firefighters (FBs), we listed as objectives ascertaining the main communicational barriers to tacit knowledge sharing and identify action strategies to be implemented by these organizations, with a view to promoting this communication.

Design / methodology / approach - A literature review on the barriers to sharing tacit knowledge, namely in its communicational dimension, was followed by a qualitative study, carried out with 8 Portuguese volunteer fire brigades, which allowed an analysis of the prevalence of these barriers and the determination of the main measures to be implemented, with a view to combating them.

Conclusions - It was possible to conclude which are the most prevalent communication barriers in these organizations and what measures to be implemented with a view to combating these barriers and promoting the sharing of tacit knowledge by increasing more effective communication.

Research limitations - This research focuses exclusively on the sharing of tacit knowledge, not considering other forms of knowledge. As this is a case study, although with very heterogeneous organizations, it cannot be replicated for different realities.

Originality / value - Studies in this area, aimed at the NPOs are scarce, as opposed to what happens in the private and public sectors. The case study option of organizations such as the Portuguese FBs, unique in their action and identity, accompanies the need, increasingly recognized by society, in enabling these organizations of competences for the best possible performance, in the face of the tragic events that have occurred in recent years in Portugal.
\end{abstract}

Keywords: communication, barriers, sharing tacit knowledge, non-profit organizations

\section{Introduction}

\subsection{Introduce the Problem}

The sharing of tacit knowledge within organizations, despite the recognition of the benefits that result from it, is not easy to establish as a regular practice. Due to their unique characteristics, the NPOs, in carrying out their missions, are increasingly promoting this sharing.

The current context of demographic changes and social, economic and cultural transformations, with repercussions in the workplace or in the organizations where individuals carry out their professional or voluntary activities, has led the authors and the organizations themselves to become increasingly more concerned with issues related to knowledge sharing. This knowledge sharing is increasingly seen as a determinant for the fulfillment of the organizations' missions.

Aware of the coexistence of two great types of knowledge, tacit and explicit, it is about the challenges that arise when sharing the first, in an organizational context, that the objectives of this study are focused on. Due to its characteristics, the process of sharing this tacit knowledge is difficult to implement, and it is possible to find in the literature a set of barriers that arise. Of a more organizational, individual or technological nature, these barriers have a common denominator, the necessary communicational process for the message to pass effectively between interlocutors.

If, on the one hand, studies focusing on the survey and impact of these communicational barriers on the NPOs are scarce, even more scarce are those that may present performance matrixes, which contain strategies, measures or 
concrete actions to combat them. Thus, it becomes a priority for the present study to understand which communication barriers arise from this sharing of tacit knowledge, regardless of their individual, organizational or technological origin and what are the ways to overcome them.

Starting with these objectives, this article presents the results of an investigation on communication barriers in Portuguese FBs, a type of NPOs that develops its activities in the area of civil protection and pre-hospital emergency. It should be noted that in Portugal there is no other type of organization in which its driving force is composed of a set of purely voluntary elements and collaborators that provide the minimum daily operational services, ensuring the volunteers the night and weekly service, with an increased intervention and availability of these elements in the summer season, with a marked decrease in service in the remaining seasons, where the hierarchical structure follows a paramilitary regime, but which may include at the top of its pyramid firefighters who only perform work within the scope of voluntary service operating in a service area as sensitive as civil protection.

Thus, the article begins with a brief introduction to the subject and with the keywords, followed by the theoretical framework, focusing on the sharing of tacit knowledge and the importance of communication for this purpose, namely, how this communication, when not well made or promoted by the organization, can constitute a type of barrier to this sharing. The next point deals with the presentation and discussion of the results, using quotes from the interviewees and confronting the literature, in relation to each identified communication barrier. Finally, we present the conclusions and recommendations for future studies.

\subsection{Describe Relevant Scholarship}

Sharing tacit knowledge can be difficult when faced with obstacles and facilitated when faced with facilitators. According to Baumard (1999), Blair (2002) and Laupase (2003), it is possible to identify obstacles to the transfer of tacit knowledge, but there are few studies that focus on the potential obstacles to the transmission of tacit knowledge in organizations with the specificity of NPOs. For this purpose, communication plays a fundamental role, being able to constitute itself as a typology of barriers (Oliveira and Pinheiro, 2020).

According to Sun et al. (2018), the communication process has four characteristics: it happens at any time; it is bidirectional, that is, we can both receive and be able to provide information to someone; it is something emotional, because the way information is transmitted influences its understanding; and it is interdependent, in that the good result of the communication depends on both the sender and the receiver.

These characteristics have led that, in an organizational context, communication has become a fundamental factor for the fulfillment of missions. Focusing on the study of organizational communication, it should be noted that its main objective is to facilitate the achievement of organizational objectives (Baker, 2002). The constant changes in information technologies, individual characteristics and organizational structure have been influencing the communication process.

According to Marques (2016), communication, when carried out effectively, favors the involvement of employees with the principles of the organization, in such a way that they end up feeling an integral part of the company's success. Which leads to the conclusion that organizational communication, then, has an essential role in the management of strategic relationships and interactions, such as knowledge sharing.

In this way, a good internal communication process can generate: greater commitment on the part of employees; superior organizational results; influence the organization's reputation; greater knowledge sharing; and greater involvement on the part of the collaborators (Verghese, 2017), so, in the context of demographic changes and social, economic, cultural transformations, with repercussions in the workplace and in organizations, the authors and the organizations themselves have come to be increasingly concerned with issues related to knowledge sharing (Cloutier, et al., 2012).

Here, it is important to note that there are two types of knowledge: explicit knowledge, which refers to data or technical information that is described in a formal language (such as in manuals) and that can be easily updated and shared within the organization. organization; and tacit knowledge, something cognitive, composed of mental models, values, beliefs, perceptions and assumptions (Smith, 2001).

According to Vij and Farooq, (2014), converting tacit knowledge into explicit knowledge is one of the biggest challenges of knowledge sharing.

Focusing on the sharing of tacit knowledge, it is possible to find in the literature three types of most prevalent barriers: individual, organizational and technological barriers.

At the individual level, for Riege (2007), barriers to knowledge sharing are generally related to factors such as 
lack of communication and social networking skills, cultural differences, overemphasis on the status of the occupied position and the lack of time and confidence. For Awad and Ghaziri (2007) and Mohajan (2016), personality, temperament, attitude, interpersonal skills, and pride about ownership or the idea of being the exclusive owner of something, are individual factors that make sharing difficult of tacit knowledge. For these authors, in some cases, individuals avoid sharing knowledge because of the risk of exposing their knowledge.

At the organizational level, it is important to mention the contributions of Mohajan (2016) that brings us to the question of the role of leaders and the strategies they adopt for their organizations. According to Joia and Lemos (2010), the reason for being a more or less bureaucratic, hierarchical or inflexible organization, which, therefore, makes it more difficult to share tacit knowledge, is often based on the decisions of its leaders. For Awad and Ghaziri (2007) and Holste and Fields (2010) the promotion of trust among its members provides an organizational environment favorable to the sharing of tacit knowledge, but this trust can be broken when individuals fail to recognize the source of that knowledge. For other authors such as Housel and Bell (2001), actions such as restructuring or downsizing also lead to a loss of valuable knowledge in organizations, or simply, when an individual who has great expertise or skill leaves the organization without said knowledge being transmitted to a potential replacement beforehand. Other organizational barriers are presented by Riege (2005), such as excessive competitiveness among employees, high staff turnover, limited access to resources that prevent the organization from recognizing or rewarding its employees, the prevalence of unilateral top-down, the lack of transparency in the organization or even the lack of highly qualified and experienced personnel.

At a technological level, according to Mohajan (2016), technology has changed the way organizations operate and how they now have the means to access and provide instant access to information and data over long distances. However, for the same author, technology is also a challenge, in the sense that it sometimes hinders the application and management of tacit knowledge. For Awad and Ghaziri (2007) and Laudon and Laudon (2012), organizations that only invest in information and communication technology systems and processes become overdependent on this technology and invest little in the individual knowledge of their employees, which can undermine tacit knowledge management. Riege (2005) also presents a set of technological barriers to the sharing of tacit knowledge, such as the insufficiency of these to respond to the tasks requested, their inadequacy in relation to the needs of the members of the organization, their inadequacy in relation to the need to communicate with elements of the organization, the complexity of its use by the elements of the organization, its inability to enhance the performance of the elements of the organization and a deficient technical support for their maintenance.

But if this may be the genesis of the barriers under study, in each of these typologies it is possible to perceive the presence and importance of communicational processes (Davenport and Prusak, 1998), so the present study will dedicate itself to understand what are the main barriers that interfere in this communicational process, making it difficult to share tacit knowledge and what to do to eliminate them.

\section{Method}

In order to pursue the objectives, it was decided to follow a qualitative methodology. One of the most frequent qualitative approaches in data processing is called content analysis. According to Bardin (2006), the content analysis procedures operate directly in the text or in the communication transcripts, being able to employ both qualitative and quantitative operations. Thus, in this investigation, the content analysis technique was used. The purpose of content analysis is, therefore, and according to Bardin (2006), to produce inferences, working with traces and indices highlighted by more or less complex procedures. Since tacit knowledge is intrinsically related to the environment in which it develops, qualitative analysis, according to Strieder (2009), should not only make it possible to capture the meaning that people attribute to phenomena, but also allow to determine values, beliefs or opinions about these same phenomena.

The unique characteristics of this type of organizations, the social, economic and demographic differences that characterize the geographical contexts in which these FBs are located, as well as the absence of previous studies, are some of the reasons that indicate a lesser knowledge of the scientific community regarding the phenomenon under study and that leads us to a first qualitative approach, using semi-structured interviews, as an incentive for the interviewees themselves to reveal their own interpretations, or even, new sources of evidence.

For this purpose, the snowball sample selection technique was followed, from among the FBs that were involved in the two major forest fires of 2017 in Portugal. For the World Health Association (1994), the snowball technique is a form of non-probabilistic sample, where the initial participants indicate new participants until the saturation point is reached, that is, when the phenomenon under study is characterized by different stakeholders without adding new relevant information to the investigation. In this context, eight interviews were carried out at the premises of the respective FBs, in places such as personal offices or meeting rooms. The interviews were recorded 
by means of audio recording, in order to store all the answers to the questions asked. For the purpose of analyzing and discussing the results, the 8 interviewees will be cited as E1, E2. E3, E4, E5, E6, E7 and E8.

\section{Results}

The first communication barrier identified in this study is insufficient or nonexistent communication. Communication is fundamental to the organization, as mentioned by Davenport and Prusak (1998) and Hendriks (1999). These authors tell us that communication can be verbal or written, while Riege (2005) argues that personal interactions are fundamental for sharing tacit knowledge, so the insufficiency or absence of communication that puts the actors in interaction reveals itself as a very important barrier.

From the testimonies collected, it was possible to verify that the majority of self-employed firefighters have other occupations or professional activities that make it impossible for them to countless times, to be present at times when the face-to-face communication process occurs and where sharing takes place:

"The main barriers that exist are related to the fact that most firefighters are volunteers. As such, they have another activity that is central to their lives. Some work, others study and that tells me that we have to adapt to the availability of each one. If availability is already low for operational activity, it is even less for tasks that involve the transmission of knowledge. There is no communication strategy that resists". E1

Internal competitiveness within FBs can also be considered a barrier to this sharing, since the organization's structure is based on a paramilitary, pyramidal, formal and relatively bureaucratic and not very flexible logic. In these contexts, where internal competitiveness is emphasized with a view to career progression, it is relatively easy to determine the prevalence of a barrier of this type. This barrier also finds an echo in the literature, namely through the contributions of Holste and Fields (2010) and Riege (2005).

In the collected interviews we found this barrier, and for this purpose, it is important to highlight the contribution of E3, which states:

"Internal competitiveness should also be mentioned, as there is the idea, with the firefighters, that it is important to move up the hierarchy and, at times, they do not have the notion of what they should really do to be better or more quickly to be promoted. The perspective that knowledge is a source of power leads them to inhibit the communication of their knowledge". E3

Following these considerations, another barrier naturally arises based on the hierarchical structure. In this regard, it is important to mention that the distance between the top and the bottom of the pyramid of the hierarchical structure of the FBs promotes a distance between people, which normally corresponds to those who have more and less experience or tacit knowledge. This distance leads to less communication, or even to its absence, impairing the sharing of tacit knowledge, confirming the results of several studies, in particular, that of Jóia and Lemos (2010). In this regard, according to Disterer (2003), hierarchical and bureaucratic organizational structures hinder communication, information sharing and, ultimately, the sharing of tacit knowledge. It turns out that, according to O'Dell and Grayson (1998), in organizations of this type, each element acts in order to achieve and enhance its own results and rewards, which discourages the exchange of experiences. Joia (2006) points out some of these organizational factors that hinder the knowledge transfer process, including a hierarchical chain of command, specialization by function, standardized procedures for each function and the non-flexible organizational structure. Thus, according to Fahey and Prusak (2001), for the transfer of tacit knowledge to occur, it is necessary that people are accessible when someone needs the knowledge they have, regardless of their hierarchical position in the organization.

In this regard, it was possible to find in these organizations, hierarchical structures that function as communication barriers, in the sense that they do not contribute to a fluent and regular communication that allows a good sharing of tacit knowledge. To portray this barrier, E8 is cited:

"The strong sense of hierarchical structure is definitely an obstacle to communication. It often happens to me and I feel it on my skin, because my firefighters may be talking or playing games when I'm not there, but when I get close, they stop. On the one hand, I didn't care if they continued, but deep down and I even like them to adopt a more formal posture, to know that there are things they cannot do in my presence. It is a matter of respect that I like to maintain". E8

The lack of communication of the benefits of tacit knowledge sharing is another communication barrier that the present study was able to determine. For Riege (2005), it is up to the organization's management or administration to communicate that the objectives or strategies involve adopting tacit knowledge sharing practices, their advantages and the respective forms of reward and recognition. The lack of this effective and efficient communication to subordinates is a barrier to sharing tacit knowledge. 
It is possible to find this barrier with the interviewees, and to illustrate this reality, we chose the contribution of E7:

"The lack of communication about the benefits of sharing knowledge is a reality and it may even be my fault. I am aware of this reality because as a commander, part of me has this responsibility to demonstrate that there are gains for everyone and for the organization we represent if we all communicate. The difficulty is that as these gains are not palpable or immediate, not only do I not remember mentioning them, nor do the firefighters interpret them in the best way. And then that's what you see. It is one more point to improve". E7

The dimension of the organization is another barrier identified in this study and which finds scientific relevance in the works of Connelly and Kelloway (2003) and Sveiby and Simmons (2002). For these authors, the size of the organization or functional departments, when too large, can hamper tacit knowledge sharing activities. Within the FBs we find organizations of different dimensions so the present barrier makes sense.

Once again, this difficulty was identified as a barrier with influence on communication leading to the sharing of tacit knowledge and as an example, we highlight the contribution of E7:

"The size of the organization is the biggest problem. It's a lot of people. Being a lot of people, the difficulty of management, what needs to be done so that everyone understands the way forward, what is intended to be transmitted, becomes more difficult. It's one thing for me to have 50 men, it's another thing to have 125 people, and that's a difficulty". E7

The current culture is another barrier that we found in the present study. It appears that the implementation of a communicational culture that favors knowledge sharing is accompanied by the need for cultural change. According to Sveiby (1997), the culture of an organization can be compared to its spirit and to what emanates from that spirit and which leads to the fulfillment of the mission, either through the focus on customer satisfaction, on obtaining profit, on the bet research and development, among others. In a logic of tacit knowledge sharing, where communication is decisive, the need for cultural change within FBs involves providing these organizations with a communicational culture that can mitigate or eliminate this barrier that was clearly identified in the present study.

This barrier is mentioned in the interviews collected and to portray it, E2's testimony is presented:

"Look, I worked so hard in this house, we put so much money here to move this FB forward, that I am sorry that one day other people will come here to the governing bodies and see that only with the radical change in attitudes, behaviors, habits and cultures it is possible to reach a higher level in our performance. Currently, we do what we can, with what we have, but I am aware that in order to provide this FB with better prepared personnel, more knowledge, they have to pay more attention to communication. But that will only come with another philosophy, it will no longer be me in charge". E2

The low rates of knowledge retention of the most experienced elements was another communication barrier identified in this study. All organizations have elements that have more experience or tacit knowledge. For Riege (2005) and Stauffer (1999), whenever there is a prolonged absence or removal of these elements from the organization to which they belong, it is a great source of tacit knowledge that is no longer available or that is irretrievably lost, so timely adoption of communication strategies can avoid these unfavorable scenarios for sharing tacit knowledge.

This is another barrier that leads us to the low rate of knowledge retention by the most experienced elements and to portray it, the contributions of E5 and E6 stand out:

"The low retention rate of knowledge of the most experienced firefighters has also been seen to be lacking. You can talk about things, but they are not recorded. We had a mechanic here who, when he went to repair the vehicles, would lock himself up inside the workshop. He does not teach anyone so that no one else could do his job and 15 days ago he came to tell me he was leaving. He was two months off and now he is leaving. And I, who never set myself up, now have a problem, which is not having a mechanic". E5

"Low knowledge retention rate for the most experienced elements is one of our main problems. Even if we study the most important events that happen here, then everything is lost when the most experienced ones leave and take what they know with them". E6

The coexistence of people with different levels of experience, training and education, according to Riege (2005), can also be considered as an obstacle to the sharing of tacit knowledge, since it can prevail for the more experienced the feeling that it is not relevant to communicate with the less experienced, because they may not understand the message, or even if the communication is established, the less experienced may have the same level of knowledge as the more experienced, questioning its importance within the organization. 
With regard to the coexistence of different levels of experience by the elements of the organization, it is worth mentioning the testimony of $\mathrm{E} 2$ and $\mathrm{E} 4$ :

"The fact that people with different levels of experience exist and live together here in FB is a difficulty because if we could be at the same level it would be much better. People with no training coexist here, with learning difficulties, people with family difficulties and problems, alcoholism problems, as we have teachers, graduates, nurses here. This lack of training and learning difficulties of some, makes the formative communication and transmission of knowledge of others difficult and without this knowledge, they hardly become experienced firefighters, so the experience levels are different and this is the reason. Basically, it is a vicious cycle". E2

"Here, too, there is a low average level of academic qualifications, being able to count the graduates with the fingers of one hand. The negative influences are those that result from the natural lack of interest in the knowledge of people who, in their own private life, also chose not to follow their studies". E4

The last communication barrier identified was the lack of time to establish contacts and increase the relationship between people. At this level, O'Dell and Grayson (1998) and Michailova and Husted (2003) also mention that the organization's managers must alert to the need and benefits of sharing tacit knowledge, the shortage of time that employees have for the most different tasks lead them to choose to do immediately what can be translated into a visible or palpable result for the organization, which is attentive to the ideal prerequisites for an effective sharing of tacit knowledge. The relationship between people and the establishment of a relationship network, even if informal, is essential for communication to flow and thus fight this barrier that is also present in FBs. For HaldinHerrgard (2000), the dissemination of knowledge comprises a set of tasks that, due to their slowness, may not be compatible with the speed experienced in the business world. In this segment, Davenport and Prusak (1998) state that in an organizational context, time is a scarce resource and more and more must be spent on tasks that bring added value to the organization. Now, for Fahey and Prusak (2001) and for Haldin-Herrgard (2000), the sharing of tacit knowledge still needs more time to become effective, since, according to Lemos and Joia (2012), this sharing results from the exchange of experiences, reflections and dialogue. For Leonard and Sensiper (1998) and Roberts (2000), this availability of time is really one of the fundamental characteristics for sharing tacit knowledge.

Regarding the lack of time for communication that leads to knowledge sharing, it is important to highlight the testimonies of $\mathrm{E} 5$ and $\mathrm{E} 6$ :

"The lack of time, whether we like it or not, is not a serious problem, because the availability of time is not what would be necessary to do things as we would like. Communicating, and mainly, communicating with quality, in order to lead someone to increase their knowledge, is something that requires time and this is, today, a scarce asset". E5

"Lack of time to identify who needs a greater sharing of knowledge, and as an example, I can refer back to the one I just gave, in which it took me about a month to identify expectations about the rewards that firefighters have. It is not easy to have effective communication with the limitations that we all have". E6

Following the identified communication barriers, the theoretical framework given to them and the collected contributions obtained from the interviewees, an action matrix is presented below, with the view to suggesting strategies, measures and concrete action actions, with the objetive of combating these barriers and promoting effective communication to promote the sharing of tacit knowledge in the organizations under study.

Table 1. Measures to be taken to combat communicational barriers in FBs

\begin{tabular}{ll}
\hline \multicolumn{1}{c}{ Barrier } & \multicolumn{1}{c}{ Measures to be taken to promote communication } \\
\hline & - Guarantee the recruitment of firefighters with adequate communication skills, in order to \\
& try to get the best out of the firefighters that the FB already has in its active staff. \\
1. Insufficient & - Provide training programs and development of communication skills appropriate to the FB \\
or non-existent & $\begin{array}{l}\text { activity. } \\
\text { communication }\end{array}$ \\
& - Support an open communication flow between all FB organizational levels. \\
& - Encourage people to be open, proactive and close, without fear of contributing ideas and \\
& opinions. \\
& - Recognize and reward well done communication. \\
\end{tabular}


- To eradicate outright the notion that holding information or knowledge is synonymous with power within the FB.

2. Internal - Introduce a reward / recognition plan to maximize tacit knowledge sharing practices.

competitiveness - Encourage or apply collective decision-making processes, where and when appropriate.

- Minimize or eliminate any personal, hierarchical or linkages to the organization that may prevent or limit tacit knowledge sharing practices.

- Reduce the application of formal power to contexts of operational intervention and disciplinary contexts and expunge it from any process of sharing tacit knowledge.

3. Hierarchical - Ensure an organic structure that supports open communication flows in all directions, structure vertically and horizontally.

- Ultimately, remove all people driven by motivational forces that do not fit into a collaborative environment and an open and tolerant organizational culture.

- Establish a sense of need to adopt measures aimed at recognition and reward, with the entire

4. Failure to organization (active body and direction of the association that oversees the FB).

communicate benefits

- Create a vision that allows a change in the performance paradigm, where recognition and reward prevail for those who share tacit knowledge, for those who seek it and for those who freely admit their mistakes and share them with the organization, avoiding its repetition by others firemen.

- Conduct a tacit knowledge audit across the FB in order to establish an effective plan that is appropriate to the size of the organization.

- Identify the greatest needs for sharing tacit knowledge, who can share what, where, how,

5. Size of the when and with whom.

organization - Identify the physical and logistical needs so that this plan for sharing tacit knowledge is carried out within normal limits.

- Assign process leaders to teams designed for this task and who promote these meetings regularly.

- Assess compliance with dimensions such as vision and mission, norms and customs, means to achieve goals, management processes, relationship with the external environment, image and reputation or any other aspect that affects the corporate culture of FB.

- Extend knowledge sharing activities to elements of the entire organization, including the

6. Current culture corporate values existing in the FB, in an attempt to change people at the expense of changing the organization as a whole.

- Incorporate the knowledge sharing culture as a part of organizational policy.

- Clearly communicate knowledge sharing policies to all firefighters, especially the newest members of the FB and incorporate this activity as part of the instruction or training to attend, for the benefit of the organization.

- Determine what really motivates people to become firefighters and then to stay in the FB.

- Communicate to the firefighters what specific impact their knowledge has on other elements and reward them accordingly.

- Ensure that firefighters are placed in positions where their responsibilities correspond to the set of skills and career aspirations they have, since incompatibilities at this level only create inefficiencies or firefighters who perform their function below what their capabilities are.

7. Low - Involve, whenever possible and beneficial, firefighters from the reserve or honor roll as knowledge mentors and/or skills trainers of the most inexperienced elements.

retention rates - Streamline mentoring and coaching programs, always involving firefighters with more experience and tacit knowledge, not exactly the most graduated.

- Offer training programs for skills and personal and operational development, internally and externally, guaranteeing and planning the succession of the current top elements of the hierarchy.

- Provide corporate benefit programs to encourage firefighters' loyalty and continuity of commitment to the FB, rewarding this loyalty through monetary and/or non-monetary incentives. 
- Design and implement guidance programs such as mentoring and coaching, through which the most experienced firefighters and not necessarily the most graduated, advise and guide firefighters with less experience and more in need of tacit knowledge sharing.

8. Coexistence - Minimize or eliminate the mentalities that may exist with people who have obtained a of people with certain level of specialization, of which they have nothing to gain from people with lower different levels levels of experience.

of experience, - Provide better integration and socialization processes among firefighters, either through the training or education promotion of these moments, or through the dynamization of spaces for this purpose.

- Remove the misperception that higher levels of education correlate with higher levels of experience and tacit knowledge.

- Remove the misconception that firefighters with low levels of formal education do not need to share or have nothing to contribute and share.

9. Shortage of - Guarantee the commitment to the sharing of tacit knowledge on the part of all the elements

time to

establish

contacts and

increase

relationships of the command and leadership structure, including the direction of the association that oversees the FB, so that structural solutions are found to overcome this problem, which may include hiring more staff for advisory services.

- Appoint a person responsible for implementing the knowledge sharing process within the organization.

\section{Discussion}

FBs are non-profit organizations with unique characteristics, where the sharing of tacit knowledge is crucial to the fulfillment of their missions. Despite the existence of several studies that mention the added value of this sharing in organizations from other sectors, studies directed at these organizations are still scarce. With the present study, it was possible to understand the main gaps in the sharing of tacit knowledge in these types of organizations, namely with regard to the main communication barriers that arise from this sharing.

With regard to these barriers, it was concluded that the most prevalent barriers in these organizations are insufficient or non-existent communication between the different members of the organization, the internal competitiveness that is felt within them, the presence of a strong hierarchical structure that cultivates the distances between the elements of different degrees or levels, the lack of communication of the benefits that can come from this sharing, the size of the organization, the current culture that does not promote an effective communication between all elements, the low rates of knowledge retention by the most experienced elements, the coexistence of people with different levels of experience, training or education and the shortage of time on the part of the different elements of the organization to establish contacts and increase relationships.

It was also possible to identify the main measures to be implemented so that the communication leads to a greater sharing of tacit knowledge, and thus, effectively bring an added value for the effective pursuit of its missions. It is a set of practical initiatives that are echoed in the literature and in the testimonies collected from the commanders of the FBs interviewed.

As a recommendation for future investigations, and in order to broaden the range of studies centered on the sharing of tacit knowledge in the NPOs, according to the lifting of the barriers that arise, it is recommended to carry out other studies aimed at personal barriers, technological, organizational or contextual, through other case studies. It is also suggested that the target organizations can be extended to other areas of NPOs, such as associations, cooperatives, private institutions of social solidarity, mutual associations, foundations, among others that are increasingly important in society.

\section{References}

Awad, E., \& Ghaziri, H. (2007). Knowledge management. Delhi: Pearson Education.

Baker, K. A. (2002). Chapter 13 - Organizational Communication. Retrieved from http://www.au.af.mil/au/awc/awcgate/doe/benchmark/ch13.pdf

Bardin, L. (2006). Análise de conteúdo, Edições 70, Lisboa.

Baumard, P. (1999). Tacit knowledge in organizations. London; Thousand Oaks, Calif.: Sage Publications. https://doi.org/10.4135/9781446217375

Blair, D. (2002). Knowledge management: hype, hope, or help? Journal of the American Society for Information Science and Technology, 53(12), 1019-1028. https://doi.org/10.1002/asi.10113 
Cloutier, E., Ledoux, É., \& Fournier, P. S. (2012). Knowledge Transmission in Light of Recent Transformations in the Workplace. Relations industrielles/ Industrial Relations, 67(2), 304-324. https://doi.org/10.7202/1009089ar

Connelly, C., \& Kelloway, E. (2003). Predictors of employees, perceptions of knowledge-sharing culture. Leadership \& Organization Development Journal, 24(5/6), 294-305. https://doi.org/10.1108/01437730310485815

Davenport, T., \& Prusak, L. (1998). Working Knowledge: How Organizations Manage What They Know, Harvard Business School Press, Boston, MA, USA. https://doi.org/10.1108/nlw.2000.101.6.282.4

Disterer, G. (2003). Fostering knowledge sharing: why and how? IADIS International Conference E-Society, 2003, Lisbon.

Fahey, L., \& Prusak, L. (1998). The eleven deadliest sins of knowledge management. California Management Review, 40(3), 265-276. https://doi.org/10.2307/41165954

Haldin-Herrgard, T. (2000). Difficulties in the Diffusion of Tacit Knowledge in Organizations. Journal of Intellectual Capital, 1(4), 357-365. https://doi.org/10.1108/14691930010359252

Hendricks, P. (1999). Why share knowledge? The influence of ICT on the motivation for knowledge sharing. Knowledge and Process Management, 16(2), 91-100. https://doi.org/10.1002/(sici)10991441(199906)6:2\%3C91::aid-kpm54\%3E3.0.co;2-m

Holste, J., \& Fields, D. (2010). Trust and tacit knowledge sharing and use. Journal of Knowledge Management, 14(1), 128-140, https://doi.org/10.1108/13673271011015615

Housel, T., \& Bell, A. (2001). Measuring and managing knowledge, McGraw-Hill Irwin, Boston.

Jóia, L. (2006). Distributive knowledge transfer processes in G2G endeavours: a heuristic frame. Knowledge Transfer for eGovernment: Seeking Better eGovernment Solutions, Verlag University, 2006. p.170-183. https://doi.org/10.4018/978-1-59904-885-7.ch103

Joia, L., \& Lemos, B. (2010). Relevant Factors for Tacit Knowledge Transfer Within Organizations. Journal of Knowledge Management, 14(3), 410-427. https://doi.org/10.1108/13673271011050139

Laudon, K., \& Laudon J. (2012). Management Information Systems: managing the digital firm. Harlow, England: Pearson Education Limited.

Laupase, R. (2003). Rewards: do they encourage tacit knowledge sharing in management consulting firms? Case studies approach. In Coakes. E. (ed.), Knowledge Management: Current Issues and Challenges (pp. 92-103). Idea Group Inc, Hershey. https://doi.org/10.4018/978-1-93177-751-3.ch008

Leonhard, D., \& Sensiper, S. (1998). The role of tacit knowledge in group innovation. California Management Review, 40(3), 112-25. https://doi.org/10.2307/41165946

Marques, J. R. (2016). A Importância da Comunicação Eficaz nas Organizações. Retrieved from http://www.unincor.br/images/imagens/2016/Informativo_novembro.pdf

Michailova, S., \& Husted, K. (2003). Knowledge-sharing hostility in Russian firms. California Management Review, 45(3), 59-77. https://doi.org/10.2307/41166176

Mohajan, H. (2016). Sharing of tacit knowledge in organizations: A Review. American Journal of Computer Science and Engineering, 3(2), 6-19.

O’Dell, C., \& Grayson, C. (1998). If only we knew what we know: identification and transfer of internal best practices. California Management Review, 40(3), 154-174. https://doi.org/10.2307/41165948

Oliveira, M. J. S. P., \& Pinheiro, P. (2020). Factors and Barriers to Tacit Knowledge Sharing in Non-Profit Organizations-a Case Study of Volunteer Firefighters in Portugal. Journal of the Knowledge Economy, 1-20. https://doi.org/10.1007/s13132-020-00665-x

Riege, A. (2005). Three-Dozen Knowledge-Sharing Barriers Managers Must Consider. Journal of Knowledge Management, 9(3), 18-35. https://doi.org/10.1108/13673270510602746

Riege, A. (2007). Actions to overcome knowledge transfer barriers in MNCs. Journal of Knowledge Management, 11(1), 48-67. https://doi.org/10.1108/13673270710728231

Roberts, J. (2000). From Know-How to Show-How: Questioning the Role of Information and Communication Technologies in Knowledge Transfer. Technology Analysis and Strategic Management, 12(4), 429-443. 
https://doi.org/10.1080/713698499

Smith, E. A. (2001). The role of tacit and explicit knowledge in the workplace. Journal of Knowledge Management, 5(4), 311-321. https://doi.org/10.1108/13673270110411733

Stauffer, D. (1999). Why people hoard knowledge. Across the Board, 36(8), 16-21.

Strieder, R. (2009). Diretrizes para elaboração de projetos de pesquisa, Joaçaba: Editora Unoesc.

Sun, H., Liu, J., \& Chen, H. (2018). Communication in Human Resource Management. Human Resources Management and Services, 1(1), 1-5.

Sveiby, K. (1997). The new organizational wealth: managing and measuring knowledge- based assets, BerrettKoehler, San Francisco. https://doi.org/10.5860/choice.35-1000

Sveiby, K., \& Simons, R. (2002). Collaborative climate and effectiveness of knowledge work. Journal of Knowledge Management, 6(5), 420-33. https://doi.org/10.1108/13673270210450388

Verghese, A. K. (2017). Internal Communication: Practices and Implications. SCMS Journal of Indian Management, 14(3), 103-113. https://doi.org/10.2139/ssrn.2690026

Vij, S., \& Farooq, R. (2014). Knowledge Sharing Orientation and Its Relationship with Business Performance: A Structural Equation Modeling Approach. IUP Journal of Knowledge Management, XII(3), 17-41. https://doi.org/10.4018/ijkm.2014070103

World Health Association (1994). Division of Mental Health, Qualitative Research for Health Programmes. Geneva: WHA.

\section{Copyrights}

Copyright for this article is retained by the author(s), with first publication rights granted to the journal.

This is an open-access article distributed under the terms and conditions of the Creative Commons Attribution license (http://creativecommons.org/licenses/by/4.0/). 